\title{
On How Event Size and Interactivity Affect Social Networks
}

Bin Xu

Cornell University

301 College Ave.

Ithaca, NY12850 USA

bx55@cornell.ed

\section{Alvin Chin}

Nokia

5 Donghuan Zhong Rd

Beijing 100176, China

alvin.chin@nokia.com

Dan Cosley

Cornell University

301 College Ave.

Ithaca, NY 14850 USA

drc44@cornell.edu
Copyright is held by the author/owner(s)

CHI 2013 Extended Abstracts, April 27-May 2, 2013, Paris, France.

ACM 978-1-4503-1952-2/13/04.

\begin{abstract}
Participating in social events or activities in the physical world is an important way for us to make new friends and build social networks. We aim to explore the role of event size and interactivity in affecting social networking behaviors. In this paper, we obtained data from an event-based social network site and conducted a quantitative analysis that reveals a relationship between online following behavior and characteristics of real-world events. We also employ behavior setting theory, social role theory, and user interview data to help us understand the quantitative results. Our finding that small events on average promote more new connections between individuals than large events has important implications for event organizers, event participants, and social media designers.
\end{abstract}

\section{Author Keywords}

Events; Friendship; User Behavior; Social Network

\section{ACM Classification Keywords}

H.5.m. Information interfaces and presentation (e.g. HCI): Miscellaneous.

\section{Introduction}

How do we make new social connections? There are many ways, for example, we use Facebook to add

recommended friends, and Twitter to follow people who 
post interesting tweets. However, much of the time, we attend events in the real world and make our new friends there. For instance, researchers attend conferences to meet other researchers, then establish new social connections by exchanging business cards and/or following them on Twitter. These events act as platforms for people to form their social networks.

We are interested in how events promote, maintain and change social networks, as well as how social networks influence the creation, diversity, participation and reminiscence of events. In this paper, as the preliminary stage of whole study, we mainly explore how an event promotes new online social connections, with an assumption that social connections in physical events will migrate to online social ties [17]. Studying this is crucial to better understand the factors influencing social network evolution in both the physical and digital worlds, and to provide guidance in designing social networking applications, recommendation systems, and social organizations [18].

There is much literature on the formation and evolution of social networks in online communities such as LiveJournal [1] or online social networking sites such as Facebook [2]. However, there exist few works on the role that an event plays in social networking behaviors. Some related works include Cranshaw et al. [3] that explore the relationship between geographical colocation and online social networking, and Xu et al. [4] that study how users' physical encounters at a conference environment affect their social behaviors.

We define an event as a set of social activities which usually happens in a physical setting, and involves community interactions within organizational structures. Following Barker's behavior setting theory [5], we postulate that structural features of events affect participants' social behavior. We choose event size (a basic structural feature of an event) and the interactivity of events as interesting characteristics to analyze. These perspectives lead to two research questions fundamental to understanding how events and online social network behavior affect each other: (1) Does the size of the event influence the social networking behaviors of people who attend the event? (2) Does the nature of the event influence social networking behaviors of attendees?

To answer these questions, we explore data from Douban (http://douban.com), a Chinese social network site where organizers can create events and users can indicate their event attendance and articulate relations with other members. We also conduct interviews with users to help us evaluate the data analytic results.

We find that event size matters in the promotion of social networking. Small events, on average, induce more new following connections for individuals than large events. We also have suggestive, but not statistically significant, quantitative evidence that the type of an event may influence social networking, and that there are interactions between size and event type. Interview responses and theories from sociology suggest processes by which these differences happen, therefore providing useful implications for designing social media and events to better connect people.

\section{Data and Method}

Dataset Description

Douban is a Chinese website that advertises real-world events and helps users extend their social networks 
through common event participation [7]. Events in Douban have 10 pre-defined types: music, film, lecture, sport, commonweal, party, travel, exhibition, drama and other ${ }^{1}$. Users can browse every event's webpage, and register as attendees and/or indicate their interest in the event. Users can also see other participants at the same event and follow them just like in Twitter [7].

We crawl Douban between October $30^{\text {th }}, 2012$ and November $6^{\text {th }}, 2012$ for events in Beijing, China, and record the event type, event participants and their follow relationships, along with their timestamps. After discarding the $5 \%$ of events that have extremely large participation (over 400) to reduce the effect of outliers and to simplify result presentation, our dataset consists of a total of 299 events, 2424 attendees, and 12873 new follow relationships. Our estimates of participation are noisy and could be refined with GPS data or "Check-in" strategy.

We use size and type to represent the structura characteristics of events [6], where the size equals the number of attendees. To represent the overall effect of the event on people's social networks, we use average network degree increment which is the average number of new follow connections each participant makes in an event. We assume that a new follow is associated with a given event if (a) that event is the most recent one which both people attended before the follow and (b) the event happened less than 7 days before the follow. Condition (a) assumes that people will be induced to follow others through interaction at a single event,

1 "Commonweal" events normally include public services, such as volunteering and charity; "other" is used when organizers cannot easily use one of the other nine event types; "Salon" events are mostly lectures. while condition (b) assumes that this inducement is limited in time. We borrow the notion of attributing behavior to the most recent event from the idea of the influence of the $k^{\prime}$ th friend in [1]; the idea of inducement having limited duration is based on the salience of the interaction with people fading as new people and events occur.

These modeling decisions are not the only possible ones: changing the duration, accounting for the influence of multiple events, or knowing the preexisting follower networks might allow us to build more nuanced models. But they are reasonable decisions that llow us to focus on constructs that are of direct interest and readily available to support these initial analyses.

Interviews about Events and Social Networking To complement the quantitative results, we also interview 7 graduate students from Beijing University of Posts and Telecommunications, 5 male, and 2 female. Their ages vary between 22 and 26, representing the age range of a large number of Douban users [7]. We conduct a semi-structured interview, covering details of their recent event participation, as well as their social networking behaviors before, during and after the events. All interviews are conducted in Chinese, in a lab room of a research institute. The interviews are also recorded under the permission of interviewees, and translated later.

\section{Analysis and Results}

Event Size and Social Networking

We first explore whether the event size affects users' following behaviors by plotting average network degree increment by event size, as shown in Figure 1 , after 
Relation between Event Size and Network Degree

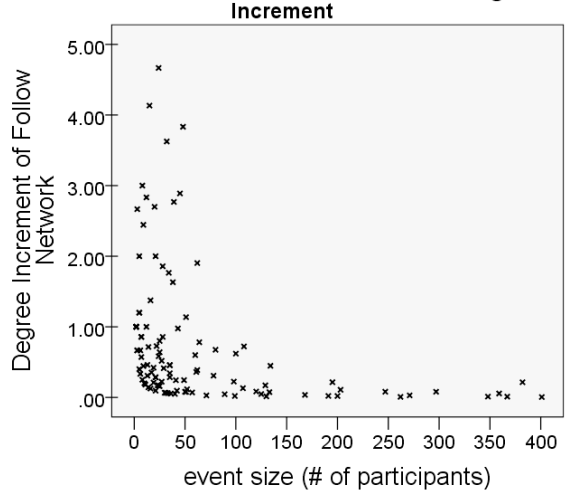

Figure 1. Relationship between event size and average network degree. discarding the very few events with extremely large participation (over 400 ) that is only $5 \%$ of total events in dataset.

With the exception of some outliers, the network degree increment tends to be higher in smaller events, the per capita level of new follower establishment in events tends to be a decreasing monotonic function of event size, which indicates that participants in small events (with event size less than 100) make more new connections on average than participants in larger events.

This is an interesting finding as this contradicts an intuitive hypothesis that there might be more potential new friends to make at a larger event, because the probability for making friends would increase when we have more candidates. Nevertheless, according to our analysis, even with limited number of candidates to connect with in small social events, people eventually manage to make more new connections. This suggests that small events are better at encouraging social networking between participants than large events. We now give theoretical speculations for this finding in three scales: individual, group, and organization.

First, interacting with others consumes individuals' attention. In large events, attention is spent across many people, leading to lower engagement per person and probability of a social connection. Interviewees' opinions also support this idea, as in this sentiment about the dynamics at a large party: "It is not easy to make a lot of new friends there, I talked with other students and professors, but I still felt we were not friends yet...I like parties having small group of people, it is easy to have long conversations".
From a group perspective, small events tend to form small groups that on average have more 2-hop links [8], which means there is a higher probability for two unlinked participants to have common connections, and thus a higher possibility of interactions between them via mutual friends [4] leading to future direct links (1hop links). In large events, however, the probability for an individual to have 2-hop links is relatively low given the number of total participants (nodes), therefore it is harder to establish new connections.

Third, size changes the "manning" structure of organizations [9]. In large organizations, the absolute number of available social positions is large, but given the number of participants, the positions are relatively scarce [10]. By contrast, small organizations have fewer positions, but the potential participants are scarce, which causes the "under-manned" phenomenon [5]. To fill the structure of organizations, small organizations must induce higher level of participation [11], and these active participants are more likely to make connections with others than passive attendees.

Event Type and Social Networking

Our second question is how different event types correlate with following behavior. For analysis, we select the top 5 labeled event types based on frequency

\begin{tabular}{|l|l|l|l|l|l|}
\hline & Salon & Music & Party & Film & C'weal $^{\prime}$ we. \\
\hline All $(\mathrm{s}<=400)$ & 0.068 & 0.097 & 0.215 & 0.042 & 0.249 \\
\hline $\mathrm{s}<=20$ & 0.215 & 0.153 & 0.024 & 0.216 & 0.454 \\
\hline $20<\mathrm{s}<=50$ & 0.045 & 0.173 & 0.475 & 0.004 & 0.209 \\
\hline $50<\mathrm{s}<=100$ & 0.020 & 0.064 & 0.062 & 0.041 & NA \\
\hline $\mathrm{s}>100$ & 0.005 & 0.036 & 0.004 & 0.002 & NA \\
\hline
\end{tabular}

Different Size and Types of Events. 
(215 salon events, 208 music events, 83 party events, 75 film events, and 55 commonweal events), as shown in Table 1.

Network Degree Increment remains the dependent variable, and we could not see statistically significant differences in follow behavior between event types based on current small dataset. Nonetheless, it is still interesting to observe that there are some fair differences when both event type (party events may induce more new social connections than film events) and in how size interacts with type (medium-sized parties seem to induce more connections than small ones).

Social role theory [13] may help us understand the causal factors of such findings. The theory claims different types of events signal particular social roles to participants, whose behavior becomes more a function of role expectations than individual characteristics. Interviewees' responses showed us that they had strong sense of what to expect at different kinds of events, which changed the level of their involvement and communications with others. For instance, commonweal event participants are most likely volunteers who need to work together to support the common cause [14], which may induce social connections. On the other hand, the role of a lecture participant is usually as listener: "I came to the lecture just for listening to that professor, I did not care about meeting other people". This quote also highlights the need to understand users' goals in the design of event spaces, sizes, and structures.

\section{Discussion and Conclusion}

Although our results are based on simple models of the mutual influence of events on networks and vice versa, they have implications for the design of both events and of social network sites that support them, and point to future work that takes a more nuanced approach toward understanding this influence.

\section{Design Implications}

Because characteristics of events such as size and type can influence social networking behavior, we propose the following implications: (1) Event organizers might consider controlling their event size to be small, or dividing a big event into smaller sections or sessions, to promote participants' social interactions. (2) For individuals, intuitions that participation in larger events can let you meet more people are wrong: going to small events might allow you to meet more friends. (3) In event-based social media design such as conference attendance support tools [16] or event-based applications such as MeetUp [15], social networking features are not equally important for small and large events. For large events, features that help participants find and manage activities and information might be more central than social networking features, unless the event naturally lends itself to creating subgroups [17]. However, for small events, effective social networking features are highly desirable as they may help people project the social networks they build in person to better expand their social networks.

Limitations and Future Work

In this paper, we assume an equivalence of offline and online social establishment and we study only the size and type of the events, neglecting other attributes like duration, location, themes, etc. We also do not attempt 
to explore other influential factors including homophily, proximity, or common friends, which are also important to understand social networking both in online and offline space. We decide to first focus on events themselves to explore the unique characteristics of event-driven social networks.

In future work, we will look into more factors together, to understand what role each of them (events, homophily, and proximity) plays based on larger or cross-platform data, how their influences overlap, and how they shape our decisions on social connections both in online and offline domain.

\section{Acknowledgments}

This work is supported by NSF grant IIS 0845351 .

\section{References}

[1] Backstrom, L., Huttenlocher, D., Kleinberg, J., and Lan, X. Group formation in large social networks: membership, growth, and evolution. In Proc. KDD 2006, ACM Press (2006), 44-54.

[2] Wilson, R.E., Gosling, S.D., and Graham, L.T. A Review of Facebook Research in the Social Sciences. Perspectives on Psych. Science, May 2012 7: 203-220.

[3] Cranshaw, J., Toch, E., Hong, J., Kittur.N., and Sadeh, N. Bridging the Gap Between Physical Location and Online Social Networks. In Proc. Ubicomp 2010, ACM Press (2010), 119-128.

[4] Xu, B., Chin, A., Wang, H., and Zhang. L. Socia Linking and Physical Proximity in a Mobile Locationbased Service. In Proc. MLBS 2011, Ubicomp 2011 ACM Press (2011), 99-108.

[5] Barker, R.G. Ecological psychology: Concepts and methods for studying the environment of human behavior. Stanford University Press (1968).
[6] Morgan, D.L, and Alwin, D.F. When Less Is More: School Size and Student Social Participation. Social Psychology Quarterly, Vol. 43, No. 2 (1980), 241-252. [7] Zhao, J.,Lui, J.C.S., Towsley, D., Guan, X., Zhou, $Y$. Empirical analysis of the evolution of follower network: A case study on Douban. In Proc. INFOCOM WKSHPS, IEEE (2011), 924-929.

[8] Kwak, H., Lee, C., Park,H., and Moon. S. What is Twitter, a social network or a news media. In Proc. WWW2010. ACM Press (2010), 591-600.

9] Lawrence, P. R., \& Lorsch, J. W. Organization and Environment. Cambridge, Mass (1967).

[10] Barker, R., and Gump, P. Big School, Small School. Stanford: Stanford University Press (1964).

[11] Kleinert, E. J. Effects of high school size on student activity participation. NASSP Bulletin (1969) 53:34-46.

[12] Schoggen, P. Behavior Settings: A Revision and Extension of Roger G. Barker's Ecological Psychology. Stanford University Press (1989).

[13] Biddle, B. J. Recent development in role theory. Annual review of sociology (1986), 67-92.

[14] Reuben,, T. S., and Mackinnon, J. R. Predicting success in volunteer community service. Psychological reports (1993), 73(3), 815-818.

[15] http://www.meetup.com. December 2012.

[16] Chin, A., Xu, B., Wang, H., Chang, L. Connecting People Through Physical Proximity and Physical Resources at a Conference. ACM TIST (2012).

17] Liu, X., He, Q., Tian, Y., Lee, W. C., McPherson, J. and Han, J. Event-based social networks: linking the online and offline social worlds. In Proc. ACM SIGKDD 2012, ACM Press (2012), 1032-1040.

[18] Chin, A., Xu, B., Wang, W., Wang, X., Liu, Z. Ephemeral Social Networking: From Online Social Networking to Mobile Social Networking. Chinese Computer Federation journal. Accepted 